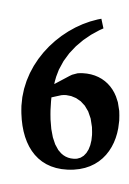

\title{
CONOCIMIENTO MATEMÁTICO SOBRE NÚMEROS Y OPERACIONES DE LOS ESTUDIANTES DE MAGISTERIO
}

\author{
(MATHEMATICAL KNOWLEDGE OF NUMBERS AND OPERATIONS \\ IN SPANISH FUTURE PRIMARY TEACHERS)
}

Araceli Gutiérrez Gutiérrez ${ }^{1}$

Pedro Gómez ${ }^{2}$

Luis Rico ${ }^{1}$

${ }^{1}$ Universidad de Granada, ${ }^{2}$ Universidad de los Andes

DOI: $10.5944 / e d u c X X 1.15581$

Cómo referenciar este artículo/How to reference this article:

Gutiérrez-Gutiérrez, A.; Gómez, P. y Rico, L. (2016). Conocimiento matemático sobre números y operaciones de los estudiantes de Magisterio. Educación XX1, 19(1), 135-158, doi:10.5944/ educXX1.15581

Gutiérrez-Gutiérrez, A.; Gómez, P.,\& Rico, L. (2016). Conocimiento matemático sobre números y operaciones de los estudiantes de Magisterio [Mathematical knowledge of numbers and operations in spanish future primary teachers]. Educación XX1, 19(1), 135-158, doi:10.5944/educXX1.15581

\section{RESUMEN}

En este artículo caracterizamos el conocimiento matemático sobre números y operaciones que los futuros profesores de primaria españoles manifestaron en el estudio internacional TEDS-M (Teacher Education and Development Study in Mathematics). A partir de los cuestionarios utilizados en ese estudio, establecemos los conocimientos que deben ponerse en juego para responder correctamente las preguntas que evalúan el dominio conceptual de números y aquellos otros que están implicados cuando se responde incorrectamente. Organizamos los resultados atendiendo al tipo de problema numérico planteado, al nivel curricular del contenido matemático implicado y al dominio cognitivo evaluado en cada pregunta. Encontramos que los futuros maestros mostraron tener un conocimiento suficiente de los contenidos previstos para primaria y los primeros cursos de secundaria con excepción del trabajo con los conceptos razón/proporción/porcentaje y la traducción de una resta de fracciones sencillas en problemas verbales. Los futuros maestros manifestaron un conocimiento insuficiente de los contenidos de matemáticas avanzadas, aunque revelaron conocer la propiedad de la densidad del conjunto de los números racionales. Por la implicación que la formación inicial en Matemáticas de los maestros tiene en la alfabetización numérica de los 
estudiantes, estos resultados son de utilidad para el diseño de las asignaturas del Grado de Maestro de Primaria en España.

\section{PALABRAS CLAVE}

Educación primaria; Formación inicial de profesores; Números; Profesores de Matemáticas; TEDS-M.

\section{ABSTRACT}

In this paper we characterize the mathematical knowledge of numbers and operations that Spanish future primary teachers showed in TEDS-M 2008 (Teacher Education and Development Study in Mathematics). We analyzed their answers from TEDS-M 2008 future teachers' questionnaires in order to establish the knowledge that was necessary to correctly answer the questions that assessed the conceptual domain of numbers and the knowledge involved when they were answered incorrectly. We classified the results based on the type of numerical problem posed, the level of curricular content involved and the cognitive domain assessed by each question. We found that Spanish future primary teachers had sufficient knowledge of the primary and lower secondary content questions with the exception of working with the concepts of reason / proportion / percentage and the translation of a simple subtraction of fractions into a word problem. Even though they reported knowing about the density of irrational numbers, Spanish future teachers showed insufficient knowledge of advanced mathematics content. Since teachers' mathematical knowledge influences the numerical literacy of students, these results can be useful for the design of the syllabi of the Primary Teacher Grade in Spain.

\section{KEY WORDS}

Primary Education; Pre-service Teacher Education; Numbers; Mathematics Teachers; TEDS-M.

\section{INTRODUCCIÓN}

\section{Justificación del estudio}

Este trabajo es un estudio secundario elaborado a partir de los resultados de TEDS-M 2008 (Teacher Education and Development Study in Mathematics) en el que profundizamos en el conocimiento matemático que manifestaron los estudiantes de Magisterio españoles sobre el dominio de números y operaciones. Partimos de que evaluar el conocimiento de los 
maestros ${ }^{1}$ en formación es importante por dos razones: primero, porque el conocimiento en matemáticas de los profesores influye en el de los estudiantes (Baumert et al., 2010; Hill, Rowan \& Ball, 2005); y segundo, porque el conocimiento que esos maestros han adquirido hasta su último año de formación puede servir como indicador del éxito que van a tener en su tarea educativa como profesores (Tatto, Sharon, Senk, Ingvarson \& Rowley, 2012).

El estudio internacional Trends in Mathematics and Science Study (TIMSS) en sucesivas aplicaciones, ha puesto de manifiesto las diferencias y deficiencias en el rendimiento matemático de los escolares de distintos países. Un factor importante que puede explicar esas diferencias tiene que ver con la variedad de aproximaciones a la formación inicial del profesorado de matemáticas en esos países. Hasta el año 2008, existían datos sobre esta diversidad desde diferentes perspectivas (Britton, Paine, Pimmyy Raizen, 2003; European Commission, 2008; OCDE, 2005; Tatto, Nielsen, Cummings, Kularatna y Dharmadasa, 1993), pero no se disponía de evidencias razonablemente completas y comparables de las diferencias existentes. TEDS-M trató de sistematizar e interpretar tales diferencias (Tatto et al., 2012).

El estudio TEDS-M respondía al interés internacional por la formación inicial de los futuros profesores de matemáticas de educación primaria y de los primeros cursos de educación secundaria obligatoria. Fue llevado a cabo por la Asociación Internacional para la Evaluación del Rendimiento Educativo (IEA, por sus siglas en inglés), pionera en la realización en este tipo de estudios desde los años 60. TEDS-M fue el primer estudio internacional comparativo que abordó la formación inicial de profesores de matemáticas y los resultados que se obtienen con esa formación.

El interés por la profundidad del conocimiento matemático y de su enseñanza que logran los maestros al final de su programa de formación, se ve avalado con la publicación del informe español por el Instituto Nacional de Evaluación Educativa (INEE) con los resultados del último estudio internacional TIMSS -2011-, en el que los alumnos de $4 .^{\circ}$ de primaria españoles obtuvieron 482 puntos en matemáticas, por debajo de la media internacional fijada en 500 puntos (INEE, 2012a). Este resultado es similar al obtenido por los futuros maestros para conocimientos matemáticos (481) en el estudio TEDS-M - también con media internacional de 500 puntos(INEE, 2012b). Los alumnos de $4 .^{\circ}$ de primaria españoles obtuvieron en TIMSS resultados de 487 puntos en el bloque de números, 476 puntos en el de formas y mediciones geométricas y 479 puntos en el de representación de datos. Estos resultados suscitan la necesidad de indagar sus causas y tomar decisiones. 
La elección del bloque de contenidos de números se debe a la importancia de la alfabetización numérica de los escolares, que se recoge en el currículo español de primaria del Ministerio de Educación y Ciencia (MEC, 2007) y a la presencia completa de este bloque de contenidos en el currículo común español de formación inicial de maestros (Rico, Gómez y Cañadas, 2014). Puesto que «la función más interesante de la evaluación educativa reside en la posible mejora que se pueda producir en el objeto evaluado como consecuencia de las decisiones que dicha evaluación suscite» (Tourón, 2009, p.127), en este estudio interpretamos la evaluación de los conocimientos matemáticos de los futuros maestros en el dominio de números desde la perspectiva de sus posibles implicaciones en la formación numérica de los alumnos de primaria.

\section{Participación española en el estudio TEDS-M}

España participó en el estudio, junto con otros 17 países, para evaluar la formación inicial de los maestros en matemáticas y obtener una información que permitiera caracterizar el conocimiento matemático y didáctico con el que los futuros maestros terminaban sus estudios, estableciendo relaciones entre ese conocimiento y las características del plan de estudios en el que habían recibido su formación. La coordinación global del estudio TEDS-M en España correspondió a la Secretaría de Estado de Educación y Formación Profesional del Ministerio de Educación, a través del Instituto de Evaluación y la dirección de la investigación a la Universidad de Granada (INEE, 2012b).

Los futuros maestros que participaron seguían el programa de formación establecido por el Real Decreto 1440/1991 (MEC, 1991), previo al actual título de Grado. La formación del maestro era generalista, tenía una duración de tres años y el profesorado español en formación recibía escasa preparación en matemáticas y su didáctica (Rico, Gómez y Cañadas, 2014). En el momento en que España participó en el estudio TEDS-M, año 2008, las universidades españolas se encontraban en un proceso de revisión y diseño de los nuevos planes de estudio para la titulación del Grado de Maestro de Primaria, dentro del proceso de convergencia al Espacio Europeo de Educación Superior. Por ello, los resultados de este trabajo pueden ser de utilidad en el proceso actual de diseño e implementación de las asignaturas del Grado de Maestro de Primaria.

\section{MARCO CONCEPTUAL}

Para plantear y seleccionar las preguntas sobre conocimiento matemático, TEDS-M se sirvió del marco conceptual elaborado para TIMSS 2007. 
Desde sus inicios, el esquema y los procedimiento del estudio TIMSS se consolidaron como válidos para evaluar el conocimiento matemático de los escolares (Garden et al., 2006; Mullis et al., 2007). La solidez del marco viene avalada por el uso reiterado que se ha hecho del mismo, a nivel nacional e internacional, como es el caso de las pruebas de evaluación general del sistema educativo español llevadas a cabo por el INEE para educación primaria (2007) en el área de matemáticas (INEE, 2012c). Puesto que nuestra investigación se basa en los instrumentos de recogida de información (cuestionarios) y en los resultados obtenidos en TEDS-M, nos servimos en parte de los criterios de ese marco conceptual para evaluar el conocimiento matemático de los futuros maestros en el dominio de números.

Como se realiza en TIMSS, las preguntas elegidas para la evaluación del conocimiento matemático de los futuros profesores de primaria que participaron en TEDS-M, atienden a una dimensión conceptual y a una dimensión cognitiva. Para la dimensión conceptual del conocimiento matemático, se consideraron cuatro bloques de contenidos o dominios conceptuales: números y operaciones; geometría y medida; álgebra y funciones; y datos y azar. En cuanto a la dimensión cognitiva, TEDS-M clasificó las preguntas según tres dominios cognitivos: conocimiento, aplicación y razonamiento (INEE, 2012b).

En este estudio pretendemos precisar el conocimiento de los futuros maestros sobre el dominio de números. Por tanto, tuvimos en cuenta la clasificación de las preguntas realizadas por TEDS-M para este dominio y realizamos además un análisis de cada una de ellas, en el que abordamos las cuatro cuestiones siguientes.

a) Identificamos el tipo de problema numérico que se planteaba, comprobando que se trataba de problemas matemáticos recogidos en la literatura de resolución de problemas.

b) Establecimos los conocimientos y capacidades matemáticos necesarios para responderla correctamente, y conjeturamos acerca de aquellos conocimientos que podrían estar implicados cuando se responde incorrectamente. En este apartado comprobamos, al profundizar en los estudios existentes, si las dificultades que percibimos en la formación de los maestros en formación son propias también de los escolares y si existían o no estudios de investigación acerca de las dificultades o errores en los que pueden incurrir los maestros en formación en dicho campo.

c) Recogimos las implicaciones didácticas de la falta de conocimiento matemático sobre un tema - justificadas con base en la literatura-. 
d) Establecimos el nivel curricular de los contenidos matemáticos evaluados según la normativa vigente en España en el momento del estudio (MEC, 2007; MECD, 2004). Abordamos la caracterización de los niveles curriculares de los contenidos según los siguientes criterios.

- Básico: este nivel incluye aquellos contenidos previstos en el currículo español de primaria.

- Intermedio: este nivel incluye los contenidos previstos en los tres primeros cursos de secundaria.

- Avanzado: este nivel incluye los contenidos previstos a partir del cuarto curso de secundaria.

Mediante esta clasificación de los contenidos matemáticos distinguimos aquellos contenidos que se presentan en el currículo de primaria de aquellos otros correspondientes a niveles superiores a los que el maestro en formación tendrá que enseñar en un futuro. De hecho, el cuestionario TEDS-M se elaboró con la intención de evaluar el conocimiento sobre los contenidos matemáticos que los futuros profesores van a enseñar, junto con otros contenidos matemáticos que se trabajen dos años más allá del nivel en que los futuros profesores van a enseñar, incluyendo también preguntas de matemáticas avanzadas (Tatto et al., 2008). Dada la diversidad de países participantes y, por tanto, la variedad de tipos de programas de formación inicial de profesores y de propuestas curriculares de primaria, hemos querido precisar para el caso español el nivel curricular del contenido al que se refiere cada una de las preguntas del cuestionario. Esta clasificación caracteriza los conocimientos matemáticos manifestados por los futuros maestros atendiendo a los niveles curriculares del contenido propuestos.

No incluimos en este apartado el marco conceptual que utilizamos para el análisis de cada una de las preguntas estudiadas. Dada la extensión que se necesita para el estudio de cada pregunta, nos limitamos a presentar más adelante un ejemplo del marco conceptual y del procedimiento de análisis de una de las preguntas. En la última parte del artículo resumimos e interpretamos los resultados obtenidos con base en el análisis de todas las preguntas del dominio de números.

\section{OBJETIVO Y MÉTODO DE LA INVESTIGACIÓN}

El objetivo general de este trabajo es caracterizar el conocimiento matemático manifestado en el estudio TEDS-M por los futuros maestros de primaria en el dominio de números. Se trata de una investigación de tipo 
descriptivo cuantitativo, a partir de datos procedentes del cuestionario del estudio internacional TEDS-M.

\section{Población y muestra}

El estudio TEDS-M definió un esquema de muestreo aleatorio, dependiente del tamaño que, en el caso de España, sirvió para seleccionar 50 instituciones de formación sobre un total de 73 que ofrecían formación inicial a futuros maestros de primaria. Dos de ellas declinaron la invitación a participar. Los procedimientos estadísticos del estudio TEDS-M establecían que la muestra aleatoria de maestros en formación debía incluir a 30 sujetos de cada institución —o a la población completa si su tamaño era inferior a 30- que en el año 2008 se encontraran cursando su último año de formación; 1093 futuros maestros respondieron el cuestionario (INEE, 2012b).

\section{Fuentes de información}

Este estudio se basa en las respuestas de los futuros maestros al cuestionario de TEDS-M2. Este cuestionario constaba de 70 preguntas que evaluaban los conocimientos de los futuros profesores, de las cuales, dos tercios correspondían al conocimiento matemático y un tercio al conocimiento didáctico. El conocimiento matemático de los futuros profesores en el dominio de números se midió con base en 10 preguntas. En la tabla 1 identificamos las preguntas de este dominio en función del tipo de problema numérico, el dominio cognitivo asignado por TEDS-M y el nivel curricular del contenido al que se refieren, y las numeramos consecutivamente.

Tabla 1

Preguntas para evaluar el conocimiento matemático sobre números

\begin{tabular}{clcc}
\hline Pregunta & Tipo de problema numérico & $\begin{array}{c}\text { Dominio } \\
\text { cognitivo }\end{array}$ & $\begin{array}{c}\text { Nivel } \\
\text { curricular }\end{array}$ \\
\hline 1 & $\begin{array}{l}\text { Proporcionalidad directa entre } \\
\text { magnitudes }\end{array}$ & Aplicación & Intermedio \\
\hline 2 & $\begin{array}{l}\text { Cantidad de números decimales } \\
\text { que hay entre dos números deci- } \\
\text { males }\end{array}$ & Conocimiento & Avanzado \\
\hline
\end{tabular}




\begin{tabular}{|c|c|c|c|}
\hline Pregunta & Tipo de problema numérico & $\begin{array}{l}\text { Dominio } \\
\text { cognitivo }\end{array}$ & $\begin{array}{l}\text { Nivel } \\
\text { curricular }\end{array}$ \\
\hline 3 & $\begin{array}{l}\text { Distinción entre números raciona- } \\
\text { les e irracionales }\end{array}$ & Conocimiento & Avanzado \\
\hline 4 & $\begin{array}{l}\text { Identificación de las propiedades } \\
\text { de las operaciones aritméticas que } \\
\text { permiten justificar una identidad }\end{array}$ & Conocimiento & Intermedio \\
\hline 5 & $\begin{array}{l}\text { Representación gráfica de fracción } \\
\text { como partes de un todo }\end{array}$ & Conocimiento & Básico \\
\hline 6 & $\begin{array}{l}\text { Traducción de una resta de fraccio- } \\
\text { nes en el enunciado de un proble- } \\
\text { ma verbal }\end{array}$ & Aplicación & Intermedio \\
\hline 7 & $\begin{array}{l}\text { Propiedades del máximo común di- } \\
\text { visor y del mínimo común múltiplo } \\
\text { de dos números }\end{array}$ & Conocimiento & Avanzado \\
\hline 8 & $\begin{array}{l}\text { Relaciones de proporcionalidad } \\
\text { entre dos cantidades de un todo }\end{array}$ & Razonamiento & Intermedio \\
\hline 9 & $\begin{array}{l}\text { Reconocimiento de razonamientos } \\
\text { sobre proporción en términos de } \\
\text { razón y porcentaje }\end{array}$ & Aplicación & Intermedio \\
\hline 10 & $\begin{array}{l}\text { Interpretación de los datos de una } \\
\text { tabla en términos de razón y pro- } \\
\text { porción }\end{array}$ & Conocimiento & Intermedio \\
\hline
\end{tabular}

Los responsables de TEDS-M liberaron los enunciados de las preguntas 3 1, 2 y 3. Las preguntas 1, 2, 4 y 5 eran de respuesta múltiple - varias opciones de respuesta de las cuales solo una era correcta-. El resto de las preguntas eran de respuesta múltiple compleja —cada opción del problema era una nueva pregunta con dos opciones de respuesta-. Mostramos a continuación dos ejemplos de problemas liberados del dominio conceptual de números. 
- Pregunta 2: Cantidad de números decimales que hay entre dos números decimales.

La Figura 1 muestra la pregunta 2. Es de respuesta múltiple y evalúa el conocimiento de los futuros maestros sobre los números decimales. Aunque la introducción de los números decimales, su ordenación y representación gráfica, así como la importancia de su uso en la vida cotidiana se encuentra en el bloque de números del tercer ciclo de primaria, consideramos que con esta pregunta se busca evaluar el conocimiento de los futuros maestros acerca de la densidad del conjunto de los números racionales y del concepto matemático de infinito, que son conocimientos propios del nivel avanzado.

¿Cuántos números decimales hay entre 0,20 y 0,30?

Marque la opción que crea correcta.
A.
9
B.
10
C.
99
D.
Un número infinito

Figura 1. Número de números decimales entre dos números decimales

- Pregunta 3: Distinción entre números racionales e irracionales.

Indique para cada número si es racional o irracional. Marque con una X la opción correcta en cada fila.
Racional
Irracional
a) $\Pi$
b) 2
c) $\sqrt{49}$
d) $\underline{-3}$

Figura 2. Pregunta sobre números racionales e irracionales

La Figura 2 muestra la pregunta 3. Es de nivel avanzado y de respuesta múltiple compleja. Evalúa el conocimiento de los futuros maestros sobre la distinción entre números racionales e irracionales. El contenido matemático de esta pregunta se contempla fundamentalmente a partir del cuarto curso de secundaria. 


\section{Procedimiento de análisis}

El método con el que abordamos el objetivo propuesto consta de los siguientes pasos:

- Análisis de cada una de las preguntas que evalúan el conocimiento matemático en el dominio de números para determinar: el tipo de problema numérico que plantea, los conceptos matemáticos que se trabajan, y los conocimientos y capacidades matemáticos implicados en sus respuestas correctas e incorrectas;

- Ubicación en la literatura de las dificultades, tanto de los escolares como de los maestros en formación, detectadas para el tema tratado en la pregunta, así como la implicación en el conocimiento didáctico de la falta de conocimiento matemático;

- Caracterización de cada pregunta en relación con el nivel curricular de su contenido matemático y el dominio cognitivo que se evalúa;

- Interpretación de los resultados de los futuros maestros para cada pregunta con base en los puntos anteriores; y

- Síntesis de la información para caracterizar e interpretar el conocimiento manifestado por los futuros maestros en el dominio de números.

Un análisis pormenorizado de las preguntas da lugar a descubrir carencias o limitaciones en el cuestionario. No obstante, en este estudio nos limitamos a estudiar los resultados que se obtuvieron con los instrumentos tal y como se aplicaron en el estudio TEDS-M.

\section{Ejemplo de análisis de una pregunta}

Presentamos, como ejemplo del método, el análisis detallado de la pregunta 1 sobre proporcionalidad directa que aparece en la Figura 3.

«Una máquina consume 2,4 litros de combustible cada 30 horas de funcionamiento. ¿Cuántos litros de combustible consumirá la máquina en 100 horas si sigue consumiendo combustible al mismo ritmo?»

Marque la opción que crea correcta.

\section{A. 7,2}


B. 8,0

C. 8,4

D. 9,6

Figura 3. Pregunta sobre proporcionalidad directa

Marco conceptual para el análisis de la pregunta

La pregunta 1 propone un problema de proporcionalidad directa entre magnitudes en un contexto de la vida cotidiana. Este contenido matemático se contempla en el primer curso de secundaria en el bloque de aritmética y álgebra como «Magnitudes directamente proporcionales. Porcentajes. Aplicación a la resolución de problemas». Este problema puede considerarse como un típico problema de regla de tres simple directa. En el problema aparecen números naturales y decimales sencillos; por tanto, se puede decir que las destrezas que los futuros profesores tendrían que poner en juego para contestar correctamente coinciden con algunos de los criterios de evaluación que se tienen en cuenta en este curso, como los siguientes.

1. Operar con números naturales y decimales y utilizarlos para resolver actividades relacionadas con la vida cotidiana.

2. Resolver problemas, eligiendo el tipo de cálculo adecuado y dar significado a las operaciones, métodos y resultados obtenidos, de acuerdo con el enunciado.

3. Emplear convenientemente la regla de tres simple directa para resolver problemas relacionados con la vida cotidiana.

Esta pregunta es de respuesta múltiple. La solución correcta es la opción $\mathrm{B}$, a la que se puede llegar, por ejemplo, realizando los cálculos que se muestran en las Figuras 4 y 5.

\begin{tabular}{c|l}
\hline Planteamiento proporcional: & $\frac{2,4}{\mathrm{a}}=\frac{30}{100}$ \\
y se resolvería & $\mathrm{a}=\frac{2,4 \times 100}{30} 8$ litros \\
\hline
\end{tabular}

Figura 4. Resolución utilizando proporciones 
Regla de tres simple directa

$\left.\begin{array}{l}2,4 \text { litros }-30 \text { horas } \\ \mathrm{a}-100 \text { horas }\end{array}\right\}$ y se resolverá $\mathrm{a}=\frac{2,4 \times 100}{30}=8$ litros

Figura 5. Resolución mediante la regla de tres simple directa

Conocimientos puestos en juego en las respuestas correctas

Para responder correctamente a esta pregunta, el futuro profesor debería tener el conocimiento matemático suficiente para reconocer que se trata de un problema de proporcionalidad directa entre magnitudes, donde hay que averiguar una cantidad desconocida que forma proporción con otras tres cantidades conocidas, así como saber qué operaciones son necesarias para resolverlo y realizarlas correctamente. Solo se valora el resultado concreto del problema; no se valoran los procedimientos llevados a cabo para lograr este resultado.

Puede haber otras vías mediante las cuales los futuros profesores hayan podido contestar correctamente, como puede ser el azar. No tendremos en cuenta esas causas para interpretar los resultados.

Conocimientos puestos en juego en las respuestas incorrectas

Con los datos disponibles, no podemos saber si los futuros profesores que contestaron de forma incorrecta manifestaron reconocer o no el problema como un problema de proporcionalidad directa entre magnitudes. Pero sí podemos identificar, dependiendo de la opción de respuesta elegida, qué tipo de cálculos hicieron y con qué datos del problema trabajaron, comprobando que los cálculos realizados no coinciden con los necesarios para resolver el problema. En este caso las posibles soluciones propuestas no proceden de utilizar todos los datos proporcionados por el enunciado:

— en la opción A, 7,2 es el resultado de multiplicar 2,4 por 3;

— en la opción D, 9,6 es el resultado de multiplicar 2,4 por 4;

- y la opción C el resultado no procede de utilizar, aparentemente, ningún dato del enunciado. 
Aunque hay estudios sobre razonamiento proporcional en maestros en formación sobre la resolución de problemas de proporcionalidad directa (por ejemplo, Valverde y Castro, 2012) no hemos entrado en ello dado que, en este caso, solo se valora el resultado concreto del problema y no los procedimientos llevados a cabo para lograr el resultado en este problema.

\section{Interpretación del conocimiento manifestado por los futuros maestros}

En la Tabla 2 presentamos la caracterización del conocimiento manifestado por los futuros maestros con base en el análisis anterior. En la primera columna, aparece el porcentaje de futuros maestros correspondiente a cada tipo de respuesta — segunda columna-. En la tercera columna, interpretamos estos resultados en términos de los conocimientos que los futuros maestros pudieron poner en juego.

Tabla 2

Caracterización del conocimiento manifestado en la pregunta 1

\begin{tabular}{cll}
\hline$\%$ & \multicolumn{1}{c}{ Respuesta } & \multicolumn{1}{c}{ Conocimientos } \\
\hline $77,4 \%$ & Correcta (B) & $\begin{array}{l}\text { Reconoció el problema de proporcionalidad } \\
\text { directa entre magnitudes y supo resolverlo } \\
\text { Operó correctamente con números decima- } \\
\text { les sencillos }\end{array}$ \\
\hline $15,7 \%$ & Incorrectas (A y D) & $\begin{array}{l}\text { No realizó los cálculos adecuados para re- } \\
\text { solver el problema } \\
\end{array}$ \\
& & $\begin{array}{l}\text { Solo utilizó uno de los datos que ofrece el } \\
\text { problema }\end{array}$ \\
\hline $6,9 \%$ & Incorrecta (C) & $\begin{array}{l}\text { El resultado no procede de los datos dados } \\
\text { por el problema }\end{array}$ \\
\hline
\end{tabular}

Los resultados de la Tabla 2 indican que el 77,4\% de los futuros maestros manifestó conocimiento matemático suficiente para reconocer el problema de proporcionalidad directa entre magnitudes, lo resolvió e identificó la solución operando correctamente con números decimales; mientras que un $22,6 \%$ no supo resolverlo, ya sea porque no realizó los cálculos adecuados, no utilizó los datos proporcionados o el resultado no procede de esos datos. Este 22,6\% tiene mayor posibilidad de no poder resolver cuestiones didácticas sobre proporcionalidad directa como se apunta en Gutiérrez, Gómez y Rico (2012). 


\section{INTERPRETACIÓN DE LOS RESULTADOS ESPAÑOLES PARA EL DOMINIO DE NÚMEROS}

Presentamos a continuación los resultados que los futuros maestros obtuvieron en el estudio TEDS-M en el dominio de números junto con su interpretación. Lo hacemos atendiendo al nivel curricular de los contenidos matemáticos.

\section{Caracterización del conocimiento manifestado en el nivel básico}

La información que presenta la Tabla 3 caracteriza el conocimiento manifestado por los futuros maestros en la única pregunta cuyo contenido matemático corresponde al nivel básico. En el primer grupo de columnas, aparece el porcentaje de futuros maestros que contestaron de forma correcta o incorrecta. En el segundo grupo de columnas, identificamos el tipo de dominio cognitivo asignado por TEDS-M. Incluimos en la última columna una descripción de estos conocimientos a partir del análisis que hemos hecho de cada pregunta.

Tabla 3

Caracterización del conocimiento manifestado en el nivel básico

\begin{tabular}{ccc}
\hline Porcentaje & $\begin{array}{c}\text { Dominio } \\
\text { cognitivo }\end{array}$ \\
\hline Co I & C A R & \multicolumn{1}{c}{ Descripción } \\
\hline Representación gráfica de fracción como partes de un todo \\
\hline $76,3 \%$ & $\sqrt{ }$ & $\begin{array}{l}\text { Conocía la representación gráfica de fracción } \\
\text { como partes de un todo }\end{array}$ \\
\hline $23,7 \%$ & $\sqrt{ }$ & $\begin{array}{l}\text { No conocía la representación gráfica de fracción } \\
\text { como partes de un todo }\end{array}$ \\
\hline
\end{tabular}

Nota: $\mathrm{Co}=$ porcentaje de respuestas correctas; $\mathrm{I}=$ porcentaje de respuestas incorrectas; $\mathrm{C}=$ conocimiento; $\mathrm{A}=$ aplicación; $\mathrm{R}$ = razonamiento

Como vemos en la Tabla 3, el 76,3\% de los futuros maestros de la muestra manifestó conocer la representación gráfica de fracción como partes de un todo. Los números fraccionarios aparecen a partir del segundo ciclo de primaria para expresar partes de la unidad y relaciones entre cantidades en contextos reales. También se contempla en este mismo ciclo su representación gráfica mediante gráficos simples o en la recta numérica y se profundiza en esto en el tercer ciclo. 


\section{Caracterización del conocimiento manifestado en el nivel intermedio}

De la misma forma que lo hemos hecho en la Tabla 3, presentamos en la tabla 4 la caracterización del conocimiento manifestado por los futuros maestros en las 6 preguntas cuyo contenido matemático corresponde al nivel intermedio. Cada bloque de filas de resultados se refiere al tipo de problema numérico considerado. Se incluye la información de la Tabla 2 por corresponder a contenidos del nivel intermedio.

Tabla 4

Caracterización del conocimiento manifestado en el nivel intermedio

\begin{tabular}{|c|c|c|}
\hline Porcentaje & $\begin{array}{l}\text { Dominio } \\
\text { cognitivo }\end{array}$ & \\
\hline Co I & C A R & Descripción \\
\hline \multicolumn{3}{|c|}{$\begin{array}{l}\text { Identificar las propiedades de las operaciones aritméticas que permiten } \\
\text { justificar una identidad }\end{array}$} \\
\hline $29,1 \%$ & $\sqrt{ }$ & $\begin{array}{l}\text { Identificó las propiedades de las operaciones arit- } \\
\text { méticas que permiten justificar una identidad }\end{array}$ \\
\hline $70,9 \%$ & $\sqrt{ }$ & $\begin{array}{l}\text { Identificó propiedades de las operaciones aritmé- } \\
\text { ticas que no justificaban la igualdad y que no se } \\
\text { podían concluir a partir del problema }\end{array}$ \\
\hline
\end{tabular}

Interpretación de los datos de una tabla en términos de razón y proporción

\begin{tabular}{|c|c|c|}
\hline $34,3 \%$ & $\sqrt{ }$ & $\begin{array}{l}\text { Fue capaz de identificar en todos los casos si distin- } \\
\text { tas interpretaciones de una tabla eran precisas }\end{array}$ \\
\hline \multirow[t]{2}{*}{$65,7 \%$} & $\sqrt{ }$ & $\begin{array}{l}\text { No fue capaz de identificar en todos los casos si dis- } \\
\text { tintas interpretaciones de una tabla eran precisas }\end{array}$ \\
\hline & \multicolumn{2}{|r|}{ Proporcionalidad directa } \\
\hline $77,4 \%$ & $\sqrt{ }$ & $\begin{array}{l}\text { Reconoció y supo resolver el problema de propor- } \\
\text { cionalidad directa entre magnitudes } \\
\text { Operó correctamente con números decimales sen- } \\
\text { cillos }\end{array}$ \\
\hline $15,7 \%$ & $\sqrt{ }$ & $\begin{array}{l}\text { No realizó los cálculos adecuados para resolver el } \\
\text { problema } \\
\text { Solo utilizó uno de los datos que ofrece el problema }\end{array}$ \\
\hline
\end{tabular}




\begin{tabular}{cll}
\hline Porcentaje & $\begin{array}{c}\text { Dominio } \\
\text { cognitivo }\end{array}$ & \\
\hline $6,9 \%$ & $\sqrt{ }$ & $\begin{array}{l}\text { El resultado no procede de los datos dados por el } \\
\text { problema } \\
\text { Traducción verbal de una resta de fracciones senci- } \\
\text { llas }\end{array}$ \\
\hline $8,49 \%$ & $\sqrt{ }$ & $\begin{array}{l}\text { Supo identificar cuáles eran las traducciones ver- } \\
\text { bales correctas de una resta de fracciones sencillas } \\
\text { con distinto denominador }\end{array}$ \\
\hline $91,5 \%$ & & $\begin{array}{l}\text { No supo distinguir las traducciones verbales co- } \\
\text { rrectas de las incorrectas de una resta de fracciones } \\
\text { sencillas con distinto denominador }\end{array}$ \\
\hline
\end{tabular}

Reconocer razonamientos sobre proporción en términos de razón y porcentaje

\begin{tabular}{|c|c|c|}
\hline $35,3 \%$ & $\sqrt{ }$ & $\begin{array}{l}\text { Distinguió los razonamientos correctos sobre } \\
\text { proporción en términos de proporción y porcen- } \\
\text { taje }\end{array}$ \\
\hline $64,6 \%$ & $\sqrt{ }$ & $\begin{array}{l}\text { No distinguió los razonamientos correctos sobre } \\
\text { proporción en términos de proporción y porcentaje }\end{array}$ \\
\hline \multicolumn{3}{|c|}{ Relaciones de proporcionalidad entre dos cantidades de un todo } \\
\hline $19,4 \%$ & $\sqrt{ }$ & $\begin{array}{l}\text { Fue capaz de identificar como verdaderas o falsas } \\
\text { afirmaciones sobre la variación de la proporción } \\
\text { entre dos cantidades de un todo }\end{array}$ \\
\hline $80,5 \%$ & $\sqrt{ }$ & $\begin{array}{l}\text { No fue capaz de identificar en todos los casos si } \\
\text { eran verdaderas o falsas afirmaciones sobre la } \\
\text { variación de la proporción entre dos cantidades de } \\
\text { un todo }\end{array}$ \\
\hline
\end{tabular}

Nota: $\mathrm{Co}=$ porcentaje de respuestas correctas; $\mathrm{I}=$ porcentaje de respuestas incorrectas; $\mathrm{C}$ = conocimiento; $\mathrm{A}=$ aplicación; $\mathrm{R}=$ razonamiento

Para la interpretación de la Tabla 4 distinguiremos los resultados para cada uno de los dominios cognitivos.

\section{Conocimiento del contenido matemático}

Como se aprecia en la Tabla 4, las preguntas que evalúan el conocimiento del contenido matemático trabajan las propiedades de las opera- 
ciones aritméticas y los conceptos de razón y proporción, estos últimos en relación con la lectura e interpretación de una tabla de valores.

Observamos que solo el $29,1 \%$ de los futuros maestros fue capaz de identificar las propiedades de las operaciones aritméticas que permitían justificar una identidad. Este tipo de conocimiento está contemplado en el primer curso de secundaria en el bloque de aritmética y álgebra donde se señala la importancia de la elaboración de estrategias de cálculo mental a partir de las propiedades de las operaciones numéricas.

En el segundo curso de secundaria, en el bloque de aritmética y álgebra, aparecen los porcentajes —cálculo de aumentos y disminuciones porcentuales-, así como las razones y proporciones numéricas. Para el caso de la interpretación de una tabla en términos de razón/proporción, solo un $34,3 \%$ de futuros maestros fue capaz de identificar en todos los casos cuándo distintas interpretaciones de la tabla eran precisas o no.

\section{Aplicación del contenido matemático}

Las preguntas que evalúan la aplicación del contenido matemático se refieren a los problemas de proporcionalidad directa, a la traducción de una resta de fracciones a problemas verbales de la vida cotidiana, y a problemas en los que se trabaja el concepto de proporción. Es decir, con estos tres problemas se evalúa la capacidad de los futuros maestros para aplicar sus conocimientos a problemas sencillos o rutinarios y los más habituales en la clase de matemáticas.

Como ya vimos, en el caso del problema de proporcionalidad directa, el $77,4 \%$ de los futuros maestros manifestó tener el conocimiento matemático suficiente para reconocer los problemas de proporcionalidad directa entre magnitudes, saber resolverlos e identificar la solución correcta de entre varias dadas.

Para el caso de la resta de fracciones sencillas con distinto denominador y su traducción verbal a problemas escolares en un contexto real, solo un $8,5 \%$ de los futuros maestros fue capaz de identificar, en todos los casos propuestos, qué traducciones verbales se corresponden con la resta de fracciones sencillas dadas. No consideramos que este contenido matemático sea propio de primaria al aparecer fracciones con distinto denominador.

Y por último un 35.3\% distinguió los razonamientos correctos de los incorrectos sobre el concepto de proporción en términos de proporción y porcentaje. 


\section{Razonamiento sobre el contenido matemático}

Solo hay una pregunta referida al dominio cognitivo de razonamiento en el dominio de números, en la que se trabaja el concepto de proporción. Como se aprecia en la Tabla 5, solo el 19,4\% de los futuros maestros fue capaz de identificar en todos los casos qué afirmaciones eran ciertas y cuáles falsas acerca de la variación de la proporción entre dos cantidades de un todo.

Caracterización del conocimiento manifestado en el nivel avanzado

De la misma forma que lo hemos hecho en las Tablas 3 y 4, presentamos en la Tabla 5 la caracterización del conocimiento manifestado por los futuros maestros en las 3 preguntas cuyo contenido matemático corresponde al nivel avanzado.

Tabla 5

Caracterización del conocimiento manifestado en el nivel avanzado

\begin{tabular}{ccc}
\hline Porcentaje & $\begin{array}{c}\text { Dominio } \\
\text { cognitivo }\end{array}$ \\
\hline CoI & CAR & \multicolumn{1}{c}{ Descripción } \\
\hline \multicolumn{2}{c}{ Cantidad de números decimales entre dos números decimales } \\
\hline $54 \%$ & $\sqrt{ }$ & $\begin{array}{l}\text { Sabía que entre dos números decimales hay infini- } \\
\text { tos números decimales }\end{array}$ \\
\hline $46 \%$ & $\sqrt{ }$ & $\begin{array}{l}\text { Manifestó que entre dos números decimales hay un } \\
\text { número finito de números decimales }\end{array}$ \\
\hline Distinción entre números racionales e irracionales \\
\hline $10 \%$ & $\sqrt{ }$ & $\begin{array}{l}\text { Distinguió, de una relación de números, los racio- } \\
\text { nales de los irracionales }\end{array}$ \\
\hline $90 \%$ & $\sqrt{ }$ & $\begin{array}{l}\text { No distinguió, de una relación de números, los } \\
\text { números racionales de los irracionales en todas las } \\
\text { ocasiones }\end{array}$ \\
\hline
\end{tabular}

Reconocer la validez de propiedades del M.C.D. y m.c.m de números naturales 


\begin{tabular}{ccl}
\hline Porcentaje & $\begin{array}{c}\text { Dominio } \\
\text { cognitivo }\end{array}$ & \\
\hline $15,6 \%$ & $\sqrt{ }$ & $\begin{array}{l}\text { Reconoció que el m.c.m. de dos números primos es } \\
\text { el producto de los dos números } \\
\text { Reconoció que el M.C.D. de dos números es menor } \\
\text { que ambos números o igual a uno de ellos } \\
\text { Reconoció que no siempre si se aumenta uno de los } \\
\text { números el m.c.m. aumenta }\end{array}$ \\
\hline $84,4 \%$ & $\sqrt{ }$ & $\begin{array}{l}\text { No reconoció como ciertas alguna de las tres pro- } \\
\text { piedades anteriores }\end{array}$ \\
\hline
\end{tabular}

Nota: $\mathrm{Co}=$ porcentaje de respuestas correctas; $\mathrm{I}=$ porcentaje de respuestas incorrectas; $\mathrm{C}=$ conocimiento; $\mathrm{A}=$ aplicación; $\mathrm{R}=$ razonamiento; $\mathrm{M} . \mathrm{C} . \mathrm{D} . \mathrm{=}$ máximo común divisor; m.c.m. = mínimo común múltiplo

Los números racionales, los irracionales y las propiedades del máximo común divisor y del mínimo común múltiplo son contenidos matemáticos que consideramos de nivel avanzado. En este caso, las tres preguntas correspondientes a este nivel se refieren al dominio cognitivo de conocimiento.

Como se aprecia en la Tabla 5, a pesar de tratarse de una pregunta de nivel avanzado, el 54\% de los futuros maestros manifestó saber que entre dos números decimales hay un número infinito de números decimales. Con respecto al conjunto de los números reales, aunque se puede introducir en tercero de secundaria, es a partir de cuarto cuando se estudia el número irracional, la iniciación al número real y la clasificación de los distintos tipos de números según sus expresiones decimales. Para este contenido matemático, solo el 10\% de los futuros maestros fue capaz de distinguir de una serie de números, los racionales de los irracionales en todos los casos. Para el caso de las propiedades del máximo común divisor y el mínimo común múltiplo solo el 15,6\% de futuros maestros supo distinguir en todas las ocasiones las afirmaciones ciertas de las falsas.

\section{CONCLUSIONES}

A raíz de los resultados españoles en matemáticas de TIMSS 2011, se han elaborado diversos estudios secundarios. En estos estudios se analiza, por ejemplo, la relación entre los resultados de los alumnos de primaria en matemáticas con determinados rasgos y características del maestro tales como su satisfacción profesional, su interacción con otros docentes o limitaciones para la enseñanza referida a sus percepciones respecto a los 
alumnos que presentan distintos problemas (Tourón, Lizasoaín, Castro y Navarro, 2012). No obstante, sin dejar de tener en cuenta estas variables, la calidad de la enseñanza se ve favorecida por factores como la cualificación y la formación del profesorado. Por consiguiente, los resultados que presentamos en este artículo destacan la importancia del suficiente y necesario conocimiento matemático de los futuros maestros para lograr una buena alfabetización matemática de sus futuros alumnos.

Rico (2000) ya apuntaba una posible causa para los bajos resultados de los maestros en matemáticas, cuando señalaba que si se compara el programa de formación inicial español con otros programas de formación de maestros especialistas de otros países, los maestros generalistas de educación primaria en España reciben una formación sensiblemente inferior en áreas que luego van a tener que enseñar, como es el caso de las matemáticas. Este resultado ha sido corroborado por Rico, Gómez y Cañadas (2014).

Por otra parte, la importancia que se da a la alfabetización numérica en el currículo de primaria español, con la presencia de tres bloques de números en primaria, no concuerda con los bajos resultados de los escolares españoles en TIMSS 2011, que están por debajo de la media de la OCDE. Nuestra hipótesis, basada en la relación entre el rendimiento de los escolares y la formación de sus profesores (Baumert et al., 2010; Hill, Rowan \& Ball, 2005), es que estos bajos resultados están relacionados con la deficiente formación de los maestros tanto en conocimientos matemáticos como didácticos. De hecho, destacamos con este trabajo que la falta de conocimiento matemático suficiente en el dominio de números podría ser causa de que los futuros maestros no pudieran abordar ciertas cuestiones didácticas, como ya se adelantó al profundizar en las preguntas de conocimiento didáctico del mismo dominio (Gutiérrez-Gutiérrez, Gómez, y Rico, 2014).

Los resultados de este estudio, en términos de los conocimientos matemáticos específicos que los futuros maestros manifestaron al responder a las preguntas del dominio de números en el cuestionario de TEDS-M, proporcionan información concreta que es de utilidad para los encargados del diseño e implementación de las asignaturas que abordan el conocimiento matemático de los futuros profesores en el Grado de Maestro de Primaria, que se encuentra en curso con motivo del proceso de convergencia al Espacio Europeo de Educación Superior.

\section{AGRADECIMIENTOS}

Este trabajo se ha realizado en el marco del Proyecto de Excelencia de la Junta de Andalucía P07-FQM03244 «TEDS-M España» y del proyecto 
EDU2009-10454 del Ministerio de Ciencia e Innovación, así como del proyecto "Procesos de aprendizaje del profesor de matemáticas en formación», EDU2012-33030 del Ministerio de Economía y Competitividad (España). Sus resultados forman parte de la tesis doctoral titulada: «Conocimiento de los maestros en formación sobre números y operaciones según el estudio TEDS-M», llevada a cabo por A. Gutiérrez-Gutiérrez, bajo la dirección de los doctores L. Rico y P. Gómez.

\section{NOTAS}

1. Como es usual en España, utilizaremos el término maestro cuando nos refiramos a los profesores de primaria españoles.

2. La base de datos internacional se encuentra en http://rms.iea-dpc.org/

3. Se encuentran en http://www.ugr.es/ tedsm/ 


\section{REFERENCIAS BIBLIOGRÁFICAS}

Baumert, J., Kunter, M., Blum, W., Brunner, M., Voss, T., Jordan, A., \& Tsai, Y.M. (2010). Teachers' mathematical knowledge, cognitive activation in the classroom, and student progress. American Educational Research Journal, 47(1), 133-180.

Britton, E., Paine, L., Pimm, D. y Raizen, S. (2003). Comprehensive teacher induction. Boston, MA: Kluwer Academic Publisher.

European Commission (2008). Levels of Autonomy and Responsibilities of Teachers in Europe. Brussels: Eurydice.

Garden, R., Lie, S., Robitaille, D. F., Angell, C., Martin, M. O., Mullis, I. V. S., Foy, P., \& Arora, A. (2006). TIMSS Advanced 2008 assessment frameworks. Chestnut Hill, MA: Boston College.

Gutiérrez, A., Gómez, P., y Rico, L. (2012). Conocimientos manifestados por los futuros maestros de Magisterio sobre didáctica de la matemática en el estudio TEDS-M. Ejemplo del análisis de una pregunta. En D. Arnau, J.L. Lupiáñez, y A. Maz (Eds.), Investigaciones en Pensamiento Numérico y Algebraico e Historia de la Matemática y Educación Matemática (pp.111-118). Valencia: Departamento de Didáctica de la Matemática de la Universitat de València y SEIEM.

Gutiérrez-Gutiérrez, A., Gómez, P., y Rico, L. (2014). Conocimiento didáctico de los estudiantes españoles de magisterio sobre números: resultados en TEDS-M. Cultura y Educación 26(2), 265-297.

Hill, H., Rowan, B. y Ball, D. (2005). Effects of teachers' mathematical knowledge for teaching on student achievement. American Educational Research Journal, 42(2), 371-406.
Instituto Nacional de Evaluación Educativa (INEE) (2012a). PIRLS-TIMSS 2011. Estudio Internacional de progreso en comprensión lectora, matemáticas y ciencias. Volumen I: Informe español. Madrid: Autor. Recuperado de: http:// bit.ly/1Aml7hY

Instituto Nacional de Evaluación Educativa (INEE) (2012b). TEDS-M. Estudio internacional sobre la formación inicial en matemáticas de los maestros. Informe español. Recuperado de: http:// bit.ly/17gDhYe

Instituto Nacional de Evaluación Educativa (INEE) (2012c). Educación Primaria 2007 Evaluación general del sistema educativo. Recuperado de: http://bit. ly/1zouNG0

Ministerio de Educación y Ciencia (MEC) (1991). Real Decreto 1440/1991, de 30 de agosto, por el que se establece el título universitario oficial de Maestros en sus diversas especialidades y las directrices generales propias de los planes de estudios conducentes a su obtención. BOE, 244, 33004-33008.

Ministerio de Educación y Ciencia (MEC) (2007). Orden ECI/2211/2007, de 12 de julio, por la que se establece el currículo y se regula la ordenación de la Educación primaria. BOE, 173, 31487-31566.

Ministerio de Educación, Cultura y Deporte (MECD) (2004). Real Decreto 116/2004, de 23 de enero, por el que se desarrolla la ordenación y se establece el currículo de la Educación Secundaria Obligatoria. BOE, 35, 5712-5791.

Mullis, I.V.S., Martin, M.O., Ruddock, G.J., O'Sullivan, C.Y., Arora, A., \& Erberber, E. (2007). TIMSS 2007 assessment frameworks. Chestnut Hill, MA: Boston College. 
OCDE (2005). Teachers matter: Attracting, developing, and retaining effective teachers. Paris: OCDE.

Rico, L. (2000). Formación y desempeño práctico en Educación Matemática de los profesores de primaria. Suma, 34, 45-51.

Rico, L., Gómez, P. y Cañadas, M.C. (2014). Formación inicial en educación matemática de los maestros de primaria en España, 1991-2010. Revista de Educación, 363, 35-59.

Tatto, M. T., Nielsen, H. D., Cummings, W. C., Kularatna, N. G., \& Dharmadasa, D. H.(1993). Comparing the effectiveness and costs of different approaches for educating primary school teachers in Sri Lanka. Teaching and Teacher Education, 9(1), 41-64.

Tatto, M. T., Schwille, J., Senk, S., Ingvarson, L., Peck, R., \& Rowley, G. (2008). Teacher Education and Development Study In Mathematics (TEDS-M): policy, practice, and readiness to teach primary and secondary mathematics. Conceptual framework. East Lansing, MI: Teacher Education and Development International Study Center, College of Education, Michigan State University.
Tatto, M. T., Sharon, J. S., Senk, L., Ingvarson, L., y Rowley, G. (2012). Policy, Practice, and Readiness to Teach Primary and Secondary Mathematics in 17 Countries. Findings from the IEA Teacher Education and Development Study in Mathematics (TEDS-M). Amsterdam: International Association for the Evaluation of Educational Achievement (IEA). Recuperado de: http:// bit.ly/1vjgeqJ

Tourón, J., Lizasoaín, L., Castro, M., y Navarro, E. (2012). Alumnos de alto, medio y bajo rendimiento en matemáticas en TIMSS. Estudio del impacto de algunos factores de contexto. En INEE (Ed.), PIRLS - TIMSS 2011. Estudio Internacional de progreso en comprensión lectora, matemáticas y ciencias. Volumen II: Informe español. Análisis secundario, 186-227. Recuperado de: http://bit.ly/1Lephgu

Tourón, J. (2009). El establecimiento de estándares de rendimiento en los sistemas educativos. Estudios Sobre Educación, 16, 127-146.

Valverde, G. y Castro, E. (2012). Prospective elementary school teachers' proportional reasoning. PNA, 7 (1), 1-18. 


\section{PERFIL ACADÉMICO Y PROFESIONAL DE LOS AUTORES}

Araceli Gutiérrez-Gutiérrez, Doctora en Educación por la Universidad de Granada en la especialidad de Didáctica de la Matemática. Profesora de secundaria y bachillerato.

Pedro Gómez, Doctor en Matemáticas (especialidad en Didáctica de la Matemática). Profesor del Centro de Investigación y Formación en Educación (CIFE) de la Universidad de los Andes, Bogotá. Editor de PNA. Formó parte del equipo investigador que realizó la participación española en el Estudio Internacional de la IEA sobre la Formación Inicial del Profesorado de Matemáticas (TEDS-M 2008).

Luis Rico Romero, Catedrático del departamento de Didáctica de la Matemática de la Universidad de Granada. Galardonado en 2012 con el XVIII Premio Andalucía de Investigación «Ibn al Jatib» por su contribución al Área de Didáctica de la Matemática. Coordinador Nacional de Investigación y responsable de la dirección científica del Estudio Internacional de la IEA sobre la Formación Inicial del Profesorado de Matemáticas (TEDS-M 2008) en España.

Dirección de los Autores: $\quad$ Araceli Gutiérrez Gutiérrez

C/ Santo Tomás de Villanueva, 36,

18004 - Granada

E-mail: araceligtgt@gmail.com

Pedro Gómez Guzmán

Carrera 1 N. $.^{\circ} 18 \mathrm{~A}-12$

Bogotá, Colombia

E-mail: argeifontes@gmail.com

Luis Rico Romero

Universidad de Granada

Dpto. de Didáctica de la Matemática

Facultad de Ciencias de la Educación

Campus Universitario de Cartuja

18071 - Granada (Granada)

E-mail: lrico@ugr.es

Fecha Recepción del Artículo: 3. Febrero. 2013

Fecha modificación Artículo: 7. Julio. 2013

Fecha Aceptación del Artículo: 9. Diciembre. 2013

Fecha Revisión para Publicación: 5. Julio. 2015 